\section{Pain relief during panretinal photocoagulation for diabetic retinopathy: a national survey}

\begin{abstract}
Aims Panretinal photocoagulation (PRP) for proliferative retinopathy is known to be painful for some people; however, few studies have explored the effects of pain on the procedure within clinical practice.

Methods This was a national survey of all Ophthalmic Units within the UK undertaken in late 2006. A questionnaire with 14 questions was developed, piloted, and sent to all NHS units in the UK.
\end{abstract}

Results A 77\% response rate was achieved. The vast majority of responses $(96 \%)$ were from doctors. A large proportion (79\%) of units see up to 20 cases a week and most patients require up to 5 sessions of PRP. Eighty-eight percent said that the procedure is painful. Once present, pain can affect the number and strength of burns delivered and indirectly increases the number of sessions required to complete the therapy. Sub-tenons anaesthesia was the most commonly used analgesic and there is some tentative evidence to support its effect. Few units have a pre-emptive analgesic protocol. Some respondents were using laser with lower exposure and reported reduced pain.

Conclusions PRP is painful; however, most units do not deliver pain relief pre-emptively. There may be a case to support the routine use of sub-tenons anaesthesia until the full effects of new laser techniques become known.

Eye (2009) 23, 2233-2237; doi:10.1038/eye.2008.421; published online 23 January 2009

Keywords: panretinal photocoagulation; proliferative retinopathy; pain; sub-tenons anaesthesia; survey
C Richardson and H Waterman

\section{Introduction}

Traditional treatment for proliferative retinopathy is panretinal photocoagulation (PRP) by argon laser therapy. Two recent studies report that sight is either maintained or improved following PRP in 61.9 and $80.5 \%$ of cases, respectively. ${ }^{1,2}$ The Diabetic Retinopathy Study Research Group (DRS, 1981) identified that best results in terms of sight maintenance are produced when a total of between 800 and 1600 laser burns of $500 \mu \mathrm{m}$ are applied to the peripheral retina in a scatter fashion. However, at least one study has reported that a large proportion of eyes (up to $40 \%$ ) treated received up to $30 \%$ less than the specified number of burns. ${ }^{3}$ Pain is the likely reason for the shortfall in the number of burns delivered. ${ }^{4,5}$

Undertreatment could lead to accelerated loss of sight, hence good pain management during the therapy might significantly affect the rate of blindness within people with proliferative diabetic retinopathy. ${ }^{6,7}$

There are several published papers exploring the efficacy of analgesics for PRP despite the fact that it has not been established within the literature that PRP using argon laser is actually painful. ${ }^{4,6-8}$ Common analgesics used in PRP are Entonox, paracetamol, various nonsteroidal anti-inflammatory drugs (NSAIDs), diazepam, and peribulbar anaesthesia. Despite this the most clinically effective analgesic is unknown. There are also a small number of reports describing the potential for pain relief using alterations in the strength/power of laser burns. ${ }^{9-11}$

The DRS report is now more than 25 years old and modifications in clinical practice alongside developments in laser equipment mean that there is a need to learn more about pain relief for PRP. The objectives of this study were to
School of Nursing Midwifery and Social Work, University of Manchester, University Place, Manchester, UK

Correspondence:

C Richardson,

School of Nursing Midwifery and Social Work, University of Manchester, University Place, Manchester, Lancashire M13 9PL, UK Tel: +0161 3067639 ; Fax: + 01613067707 . E-mail: clifford.richardson@ manchester.ac.uk

Received: 25 June 2008 Accepted in revised form: 11 December 2008 Published online: 23 January 2009 
identify: (1) if practitioners consider PRP to be painful and if pain has an effect on PRP, and (2) which analgesics are in regular use for PRP in the UK.

\section{Materials and methods}

A national survey was chosen to gain a representative sample of ophthalmologists' views of clinical practice and of variations within practice across units where different forms of PRP are likely to be practised following recent rapid expansion in technology. All NHS ophthalmology medical training units in the UK were identified through the Royal College of Ophthalmologists Directory (RCO, 2005) and all were included. Data were collected through a two-sided postal questionnaire addressed to the 'chief laser therapist' to ensure that all professionals performing laser were identified. Fourteen questions were devised initially from the literature and modified following discussion with consultant ophthalmologists and ophthalmic nurse practitioners. Questions covered all aspects of PRP, including unit activity, laser strength, number of burns, analgesia use, pain levels, and analgesic effectiveness. All questions had space for open comments and if specific comments were given in sufficient numbers these were added to the response data. The questionnaire was piloted on 10 doctors and nurses at the local regional eye hospital and was amended according to feedback, suggesting there was a need to clarify whether answers needed to relate to single eye or total treatment. The questionnaire was distributed in September 2006, and a second copy was sent 1 month later to all nonresponders.

\section{Statistical analysis}

Descriptive statistics were performed. Associations between analgesia and pain were sought by converting the responses to the question about PRP being painful into high/low scores (hardly ever, rarely and sometimes were taken to be low pain likelihood, and often and nearly always as high pain likelihood) and performing a cross-tabulation with each analgesic used.

The local medical research and the university ethics committees gave approval for the study.

\section{Results}

A total of 115 out of 149 questionnaires were returned giving a response rate of $77 \%$. Four were returned blank with one stating that PRP was not performed. The rest $(n=111)$ were included in the analysis, however, not all respondents answered all questions hence at times the denominator reported varies. Ninety-six percent of the
Table 1 Average number of cases treated per week and average number of sessions delivered to each patient to complete the treatment plan

\begin{tabular}{lc}
\hline Number of & Percentage \\
\hline Cases per week $(\mathrm{n}=111)$ & \\
$\quad<10$ & 38 \\
$11-19$ & 41 \\
$20-29$ & 14 \\
$30-39$ & 4 \\
$>40$ & 3 \\
& \\
Sessions per patient $(\mathrm{n}=109)$ & \\
$1-5$ & 84 \\
$6-10$ & 15 \\
$11-15$ & 1 \\
\hline
\end{tabular}

respondents were doctors $(62 \%$ consultant, $17 \%$ associate specialist, $5 \%$ specialist registrar, $7 \%$ staff grade, and $5 \%$ unspecified) and 4\% were nurses. Nearly all units (99\%) performed PRP routinely.

The number of cases treated per week and number of sessions per patient are shown in Table 1. Most units treat up to 20 patients per week and the majority of patients require up to 5 sessions of PRP to complete the therapy.

Patient requirement and tolerance were important in determining both the number and the strength of burns per session (Figure 1). A small number of respondents stated that the experience of the laser therapist and their preference $(4 ; 4 \%)$ was important to decide the number of burns, whereas others $(8 ; 7 \%)$ tried to keep the number of burns constant per session.

Additional factors influencing the strength of the laser burns, as identified by the open comments section, were the patient/retinal reaction, the experience of the laser operator, and factors within the retina including pigmentation.

Respondents were asked to grade the likelihood for pain during PRP. The answer to this question is found in Table 2. Overall 97 (88\%) identified that PRP could be painful, with only 13 (12\%) opting for rarely or hardly ever.

Virtually every respondent $(109,99 \%)$ identified that pain has a negative effect on the delivery of the therapy. Participants were given the option to give multiple responses for the question asking about the effect of pain on the patient and the procedure (Table 3 ).

A large majority of units $(77,67 \%)$ did not have a pain protocol and most of those $(62,80 \%)$ identified that routinely no analgesia was given for this procedure. Although not statistically significant a trend was found towards a likelihood for the respondent to state that patients have pain during PRP in those units that had a pain protocol in place $(r=-0.14 ; P=0.18)$. 

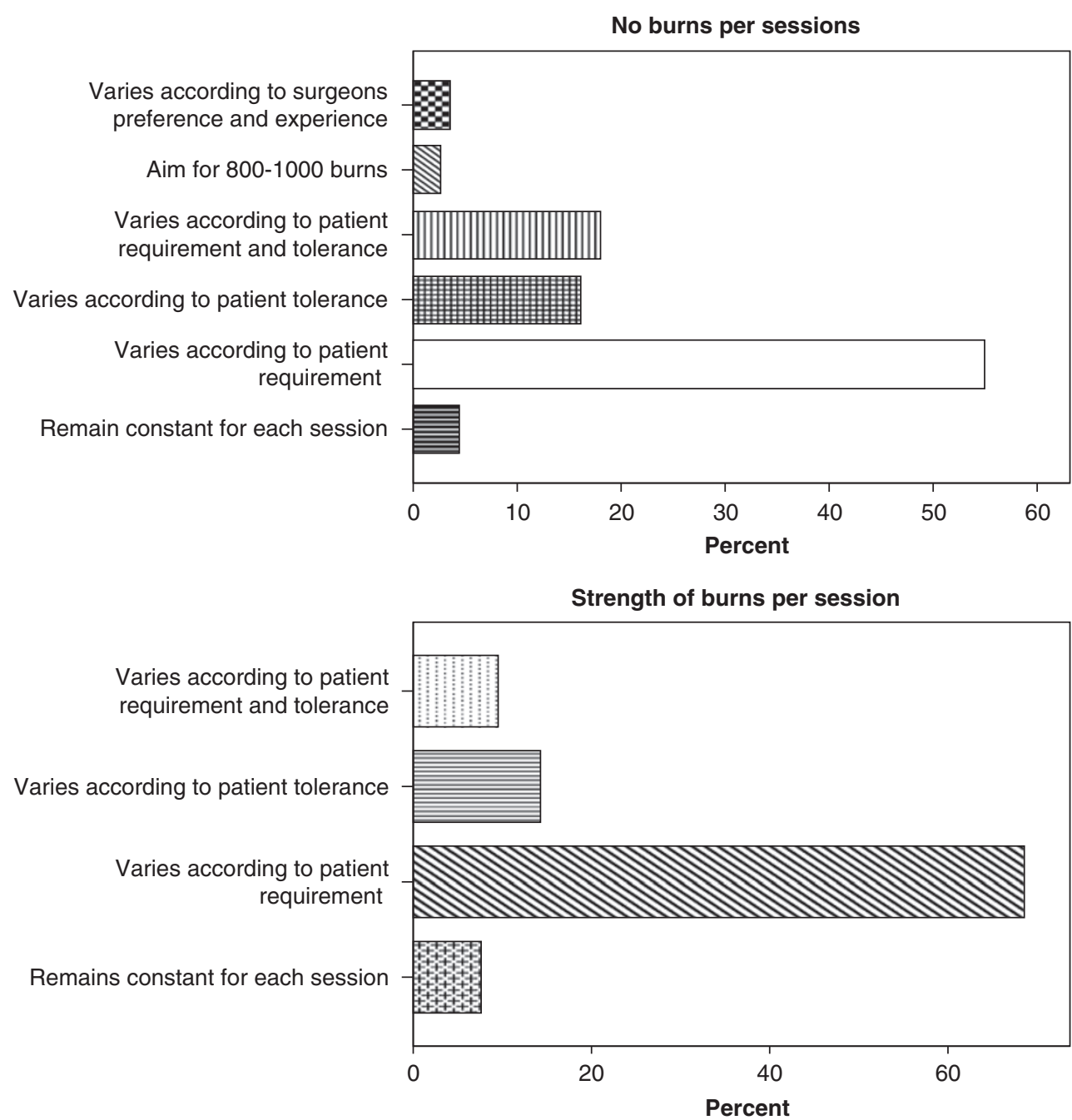

Figure 1 Number and strength of laser burns used during PRP.

Table 2 Response to question 7. Is PRP painful?

\begin{tabular}{lcc}
\hline Is PRP painful $(\mathrm{n}=110)$ & Frequency & Percentage \\
\hline Hardly ever & 5 & 5 \\
Rarely & 8 & 7 \\
Sometimes & 68 & 62 \\
Often & 21 & 19 \\
Almost always & 8 & 7 \\
\hline
\end{tabular}

Participants were asked to identify analgesics used within their unit (Table 4). The most common response was sub-tenons anaesthesia $(70,72 \%)$ and when utilized, $99 \%$ stated that analgesia was effective.

Those units that used sub-tenons $(P=0.02)$ or manipulation of the laser $(P=0.001)$ were more likely to report that PRP was not painful.
Table 3 The effect of pain on PRP

\begin{tabular}{lcc}
\hline Effect of pain & Frequency & Percentage $^{\mathrm{a}}$ \\
\hline Reduces number of burns & 89 & 82 \\
Causes distress & 86 & 79 \\
Increases number of sessions & 84 & 77 \\
Reduces strength of burns & 52 & 48 \\
Stimulates need for analgesia & 16 & 15 \\
Inadequate treatment given & 6 & 6 \\
Need to stop and start & 4 & 4 \\
Delays treatment & 4 & 4 \\
Affects compliance & 4 & 4
\end{tabular}

${ }^{a}$ Total more than 100 due to option to give multiple responses.

\section{Conclusion}

The results of the survey suggest that in late 2006 despite the fact that PRP was known and accepted by practitioners to be painful, routine use of analgesics only occurred in the 
Table 4 Routine first choice analgesia for PRP

\begin{tabular}{lcc}
\hline Analgesic $(\mathrm{n}=97)$ & Frequency & Percentage \\
\hline None & 77 & 80 \\
Sub-tenons anaesthesia & 9 & 9 \\
Oral analgesia & 4 & 4 \\
Alter laser parameters & 3 & 3 \\
Peri- or retrobulbar block & 2 & 2 \\
Entonox & 1 & 1 \\
General anaesthetic & 1 & 1 \\
\hline
\end{tabular}

minority of units. Analgesia was used in reaction to pain rather than pre-emptively. Several analgesics were used, ranging from simple oral analgesics, such as paracetamol and NSAIDs, to the use of agents traditionally used in anaesthesia, such as Entonox, local anaesthetic blocks (sub-tenons, peribulbar, and retrobulbar), and even general anaesthetics in some cases.

Sub-tenons anaesthesia was the most widely used (9\% as a first choice), but only two papers report results with this technique on small number of patients. The first tested its anaesthetic efficacy ${ }^{12}$ using 26 patients and 40 eyes, whereas the second found it was an effective analgesic in 11 out of the 12 eyes on which it was used. ${ }^{13}$

Results from this study suggest that all analgesia is effective. Earlier, however, Wu et $\mathrm{al}^{7}$ compared multiple oral and intramuscular analgesics with peribulbar anaesthesia and placebo and found that only local anaesthetics were effective at reducing pain $(P<0.0001)$. The invasiveness and potential side effects of sub-tenons and other local anaesthetic procedures may restrict their use, especially as an anaesthetised eye may be prone to accept over treatment with resultant macular oedema.

Once present, pain appeared to have a significant function in reducing the strength of burns, the number of burns, and the total amount of therapy delivered to a patient. It could also delay treatment therefore extending patient stay and a small number of respondents identified the potential for a lack of compliance with the procedure. Little or no research has investigated how the pain affects the patient. There is a need to identify the patient's experience during and even after the procedure. At present it is unknown whether the pain may continue after each treatment session and how it may influence patients' decisions to return to clinic and whether pain reduction may influence that decision. Future research is required in this important area. This is still true following the advent of the PASCAL laser that anecdotally at least appears to have less pain during the procedure, but it is unknown whether patients experience delayed effects.

Communication between therapist and patient was reported to be important in laser treatment. The partnership developed during the therapy was viewed positively and enabled the full treatment to be completed. Although this maybe true, it should not be used to the exclusion of analgesics. The use of communication has been used in other areas of pain relief and needs further exploration in this area. Laser modification/variation is also an area for future research, especially in light of the findings that light laser and short burn duration might elicit similar clinical outcomes to traditional laser. ${ }^{9,10}$

Units with pain protocols in place reported a greater likelihood for the patients to be in pain during PRP $(P=0.18)$. This is an interesting finding and may be explained by the nature of protocols. Often protocols of this kind are reactive to pain rather than pre-emptive of it. In situations where pain is an expectation as it has been with PRP, specific pain protocols are required to prevent the pain, subsequent distress, variations in treatment, and the potential for undertreatment.

These results need to be viewed with the same caution as all survey findings in that they are a report of what clinicians say they do and may not reflect what they actually do. In an attempt to elicit information as close to the clinical picture as possible, the participants were offered open comment space throughout. Many took the opportunity and the analysis was adapted to take account of them.

It was clear from these comments that in 2006 there were significant variations in treatment regimes for PRP throughout the UK, so perhaps the next step should be to re-visit the findings of the DRS (1981) taking into account the wealth of clinical experience and technological development since that time. Despite the development of medical therapies for proliferative diabetic retinopathy it is likely that PRP will continue as the mainstay of treatment, hence there is a need for standardization that should maximize the effects of this important therapy. These results suggest that this will not be possible without taking account of the pain associated with the procedure unless the new laser variations and technologies can be proven to conclusively reduce the pain and distress.

\section{References}

1 Yi Q, Bamroongsuk P, McCarty DJ, Mukesh BN, Harper CA. Clinical outcomes following laser photocoagulation treatment for diabetic retinopathy at a large Australian ophthalmic hospital. Clin Experiment Ophthalmol 2003; 31(4): 305-309.

2 Kaiser RS, Maguire MG, Grunwald JE, Lieb D, Jani B, Brucker AJ et al. One-year outcomes of panretinal photocoagulation in proliferative diabetic retinopathy. Am J Ophthalmol 2000; 129(2): 178-185.

3 Bailey CC, Sparrow JM, Grey RH, Cheng H. The national diabetic retinopathy laser treatment audit. II. Proliferative retinopathy. Eye 1998; 12(Pt 1): 77-84. 
4 Richardson C, Hollinghurst A. The use of Entonox for pain relief during argon laser therapy for proliferative diabetic retinopathy. Ophthal Nurs 1999; 3(2): 16-18.

5 Dowler JGF. Laser management of diabetic retinopathy. J R Soc Med 2003; 96: 277-279.

6 Vaideanu D, Taylor P, McAndrew P, Hildreth A, Deady JP, Steel DH. Double masked randomised controlled trial to assess the effectiveness of paracetamol in reducing pain in panretinal photocoagulation. Br J Ophthalmol 2006; 90(6): 713-717.

$7 \mathrm{Wu}$ WC, Hsu KH, Chen TL, Hwang YS, Lin KK, Li LM et al. Interventions for relieving pain associated with panretinal photocoagulation: a prospective randomized trial. Eye 2006; 20(6): 712-719.

8 Cook HL, Newsom RSB, Mensah E, Saeed M, James D, Ffytche TJ. Entonox as an analgesic agent during panretinal photocoagulation.[see comment]. Br J Ophthalmol 2002; 86(10): 1107-1108.
9 Bandello F, Brancato R, Menchini U, Virgili G, Lanzetta P, Ferrari E et al. Light panretinal photocoagulation (LPRP) vs classic panretinal photocoagulation (CPRP) in proliferative diabetic retinopathy. Semin Ophthalmol 2001; 16(1): 12-18.

10 Rimmer T, Wykes W. Quicker painless diabetic laser. Eye 2007; 21(1): 140; author reply 140-2.

11 Al-Hussainy S, Dodson PM, Gibson JM. Pain response and follow-up of patients undergoing panretinal laser photocoagulation with reduced exposure times. Eye 2008; 22(1): 96-99.

12 Friedberg MA, Palmer RM. A new technique of local anesthesia for panretinal photocoagulation. Ophthalmic Surg 1991; 22(10): 619-621.

13 Stevens J, Foss A, Hamilton A. No-needle one-quadrant sub-tenon anaesthesia for panretinal photocoagulation. Eye 1993; 7: 768-771. 